reconoce el estilo de Murillo en todo su esplendor en fechas que pueden oscilar entre 1665 y 1670. El estudio anatómico del joven es extremadamente correcto y al mismo tiempo aparece magníficamente captada la expresión de su rostro, concentrada y serena ${ }^{6}$.

Tiene, además, esta pintura claras referencias de su permanencia en España antes de salir de nuestro país, puesto que tenemos la certeza de que pasó por la colección de Godoy tal y como figura en 1850 en el catálogo de la venta de las pinturas del marqués de Montcalm en París ${ }^{7}$. En dicho catálogo de venta se describe esta pintura de la forma siguiente: Murillo l'Esclave un jeune esclave coiffe d'un turban porte de fruits dans une corbeille. D'une belle couleur et d'une execution pleine d'energie. Il a fait partie de la collection du Prince de la Paix. Aut 1 metre large $80 \mathrm{cem}$. Como puede comprobarse el cuadro es el mismo que aquí recogemos ya que sus medidas son prácticamente exactas.

Una última cuestión se nos ocurre a propósito de esta pintura y es que la Niña con flores, obra de Murillo en la Dulwich Picture Gallerie, aunque no forma pareja con esta Alegoría del Verano, sino que pertenece a otra serie pictórica, es muy posible que sea una Alegoría de la Primavera, tal y como puede observarse, puesto que ambas obras presentan claras similitudes compositivas (Fig. 2). Se trata de una joven figura femenina con un turbante sobre su cabeza en el que está prendida una rosa y que muestra un conjunto de flores en su regazo. Ambas obras están respaldadas por restos arquitectónicos a la derecha y un paisaje, aunque este detalle es casi invisible en la Alegoría del Verano ${ }^{8}$.

ENRIQUE VALDIVIELSO

Departamento de Historia del Arte de la Universidad de Sevilla

\title{
UN «DESCENDIMIENTO» DE PABLO VERONÉS Y DOS DE SU HIJO CARLETTO EN EL ESCORIAL
}

El Monasterio de El Escorial cuenta con tres «Descendimientos» catalogados por Poleró como obras de Carlo Caliari: dos en los Nuevos Museos (n. ${ }^{\circ} 85$ del cat. Poleró: L. 69,5 × 89; n. ${ }^{\circ} 189$ del cat. Poleró: L. $\left.178 \times 193\right)$ y uno en el Panteón de Infantes (n. ${ }^{\circ} 113$ del cat. Poleró: L. $272 \times 163$ ). Con el presente artículo se va a tratar de demostrar que el más pequeño debe atribuirse a su padre, Pablo Veronés, y de clarificar, en la medida de lo posible, a cuáles de ellos se refieren las menciones antiguas que se han logrado recoger.

Conviene empezar por el más pequeño que, además de ser el de más calidad, figura en

\footnotetext{
${ }^{6}$ Mide $100 \times 76 \mathrm{cms}$. Dimensiones que coinciden casi exactamente con las que don Diego Angulo recogió al referirse al cuadro de Lord Normanton.

${ }^{7}$ Catalogue de la seconde et plus important partie de la Belle Colection de tableaux de M. Le marquise de Montcalm de Montpellier, París, 25 de marzo de 1850. Agradezco enormemente a Isadore Rose-de Viejo el haberme proporcionado esta noticia que procede de su tesis doctoral: Manuel Godoy, patrón de las artes y coleccionista, Madrid, Universidad Complutense, 1983, vol. II, pp. 758-759.

${ }^{8}$ En este sentido, hemos de manifestar que nos cuesta mucho trabajo creer que esta Niña con flores de Dulwich sea un cuadro con contenido erótico tal y como manifestó J. Brown en su artículo: «Murillo, pintor de temas eróticos. Una faceta inadvertida de su obra». Goya, núms. 169-171, julio-diciembre 1982. Ciertamente nos parece impensable admitir que una persona como Murillo, fielmente vinculada a la estricta moral católica imperante en Sevilla en la segunda mitad del siglo XVII, sumamente restrictiva a la hora de aceptar representaciones pictóricas de contenido pecaminoso, pueda haber incurrido en una escena de carácter lascivo.
} 
publicaciones recientes (Fig. 1) ${ }^{1}$. El primero en mencionarlo en El Escorial pienso que sea el padre Ximénez (1764), quien lo describe en una piececita anterior a los Oratorios Reales «Sobre la puerta, un Descendimiento, de Paulo Veronés, Quadro pequeño, de gran colorido: el historiado muy devoto, y los afectos de las Figuras, con artificio y destreza:...»-, donde también lo verá Ponz: «un cuadrito historiado, de Pablo Veronés, que representa el Descendimiento de Nuestro Señor» ${ }^{2}$. Durante la invasión francesa abandonó El Escorial, ya que consta en un documento de 14 de mayo de 1813 entre los cuadros trasladados del exconvento del Rosario a la Academia de San Fernando: «N. 1.79. Vn quadro de 3 palmos y medio de alto por 4 y 3 dedos de ancho (en centímetros 73,5 × 89,4). Representa el Descendimiento del Señor; su autor Pablo Veronés, estropeado» ${ }^{3}$. Una vez de nuevo en el Monasterio, Bermejo lo menciona a nombre de Carlos Veronés en la Sacristía: «35. El descendimiento de la cruz con figuras pequeñas, por Cárlos Veronés» ${ }^{4}$, en donde continuará en tiempos de Polero ${ }^{5}$.

Ahora bien, antes de formar parte de las colecciones del Monasterio, ¿dónde se encontraba? Todo hace suponer que formara parte de la colección del marqués del Carpio y que ingresara en las colecciones reales en 1691, fecha en la que pasaron a la corona varias pinturas de dicho aristócrata como pago de lo que adeudaba a la Real Hacienda. Parece confirmar lo dicho que en el inventario de 1688 de los cuadros que el mencionado marqués poseía en el Jardín de San Joaquín figure, con el número 316 y valorado en 8.800 reales, un cuadro de Pablo Veronés de una vara y sesma de alto por una vara y cuarta de ancho $(97,5 \times 104,5 \mathrm{cms}$.), que en nota al margen se dice que se dio a Su Majestad, de «el descendimiento de la Cruz de nro. sor. q/uel le tienen nuestra señora en las Rodillas bajo de Abar y Maria Con la Sauana Tendida y nuestra señora desmayada y Santta Maria Magdalena a los pies y San Juan Teniendo a nuestra Señora y las dos Marias», y que vuelva a mencionarse en la relación de cuadros de dicho aristócrata que pasaron a la corona en $1691^{6}$.

1 Ruiz Gómez mantiene la atribución de Poleró. En cuanto a su procedencia duda en identificarlo con el «Descendimiento» citado por el padre Santos en 1667 como obra de Carlos Caliari en el altar del oratorio del prior, ya que, en su opinión, pudiera tratarse éste último del otro también hoy conservado en los Nuevos Museos. También añade que Ponz cita en 1773 otro cuadro del mismo asunto, esta vez de Pablo Veronés, en la habitación anterior a los oratorios reales. Por último, dice que con bastante seguridad puede situarse en 1820 en la Sacristía en donde lo cita Bermejo, y que corresponde al n.$^{\circ} 85$ del catálogo de Poleró. Asimismo, acertadamente relaciona su composición con el «Descendimiento» conservado en la Galleria Doria Pamphili (Catálogo de Pintura Veneciana histórica en el Real Monasterio de El Escorial, Madrid, 1991, 143-144). Por su parte, Checa — que erróneamente lo sitúa en el Panteón de Infantes — también mantiene la atribución de Poleró y lo identifica con el mencionado por el padre Santos en el altar de la Celda Alta del Prior en sus ediciones de 1657 y 1667, y que en 1681 ya se había sustituido por una escultura (Tiziano y la monarquía hispánica, 1994, p. 294). Muestra nuestro cuadrito en el ángulo inferior derecho el n. 270 en blanco, que se encuentra en parte cubierto por el número 837 en color crema, y en su ángulo inferior izquierdo parece leerse el número 85 (n. ${ }^{\circ}$ del catálogo de Poleró).

2 Ximénez, A., Descripción del Real Monasterio de San Lorenzo del Escorial, Madrid, 1764, p. 171; Ponz, A., Viage de España, Madrid, 1788, T. II, Carta V, n. 30 (Madrid, Edit. Aguilar, 1988, vol. 1, p. 440).

3 V.V., «Manuel Napoli y la colección de cuadros del exconvento del Rosario», Revista de Archivos, Bibliotecas y Museos, 1904, noviembre, p. 198.

Aunque no se puede asegurar, ya que podría tratarse de alguno de los otros dos cuadros de este asunto conservados en El Escorial, es posible que corresponda al siguiente trasladado con anterioridad, el 1 de noviembre de 1809, de El Escoria a Madrid por Quilliet: «Descendimiento, de Carlos Veronés (en caja núm. 19)» (Lasso de la Vega, M. - Marqués del Saltillo-, Mr. Frédéric Quilliet, Comisario de Bellas Artes del Gobierno Intruso (1809-1814), Madrid, 1933, p. 71 y Hempel Lipschutz, I., «El despojo de obras de arte en España durante la Guerra de la Independencia», Arte Español, 1961, pp. 241).

${ }^{4}$ Bermejo, D., Descripción artística del Real Monasterio de S. Lorenzo del Escorial y sus preciosidades despues de la invasión de los franceses, Madrid, 1820, p. 91.

5 «Veronés (Carlos)... 85 El Descendimiento de la Cruz. Nuestra Señora sostiene en su regazo el cuerpo muerto de su hijo; San Juan y las Marías le lloran. Alto, 2 piés, 7 pulg.; ancho, 3 piés, 2 pulg., 9 lín.» (Poleró, V., Catálogo de los cuadros del Real Monasterio de San Lorenzo, llamado del Escorial, en el que se comprenden los del Real Palacio, Casino del Príncipe y Capilla de la Fresneda, Madrid, 1857, p. 44-45). Curiosamente, Poleró en su «Relación de los cuadros que en otro tiempo existieron en el Monasterio de San Lorenzo el Real...» cita: «Pablo Veronés, Descendimiento de la Cruz (cuadro pequeño)», sin darse cuenta que todavía se conservaba, aunque Bermejo le hubiera cambiado la atribución (Poleró, V., op. cit., 1857, p. 187).

${ }^{6}$ Burke, M. B., Private collections of Italian art in the seventeenth Century Spain, Universidad de Nueva York, 1984 vol. II, pp. 253 y 378. Resulta difícilmente comprensible lo que quiso expresar el autor del inventario al escribir «bajo de 

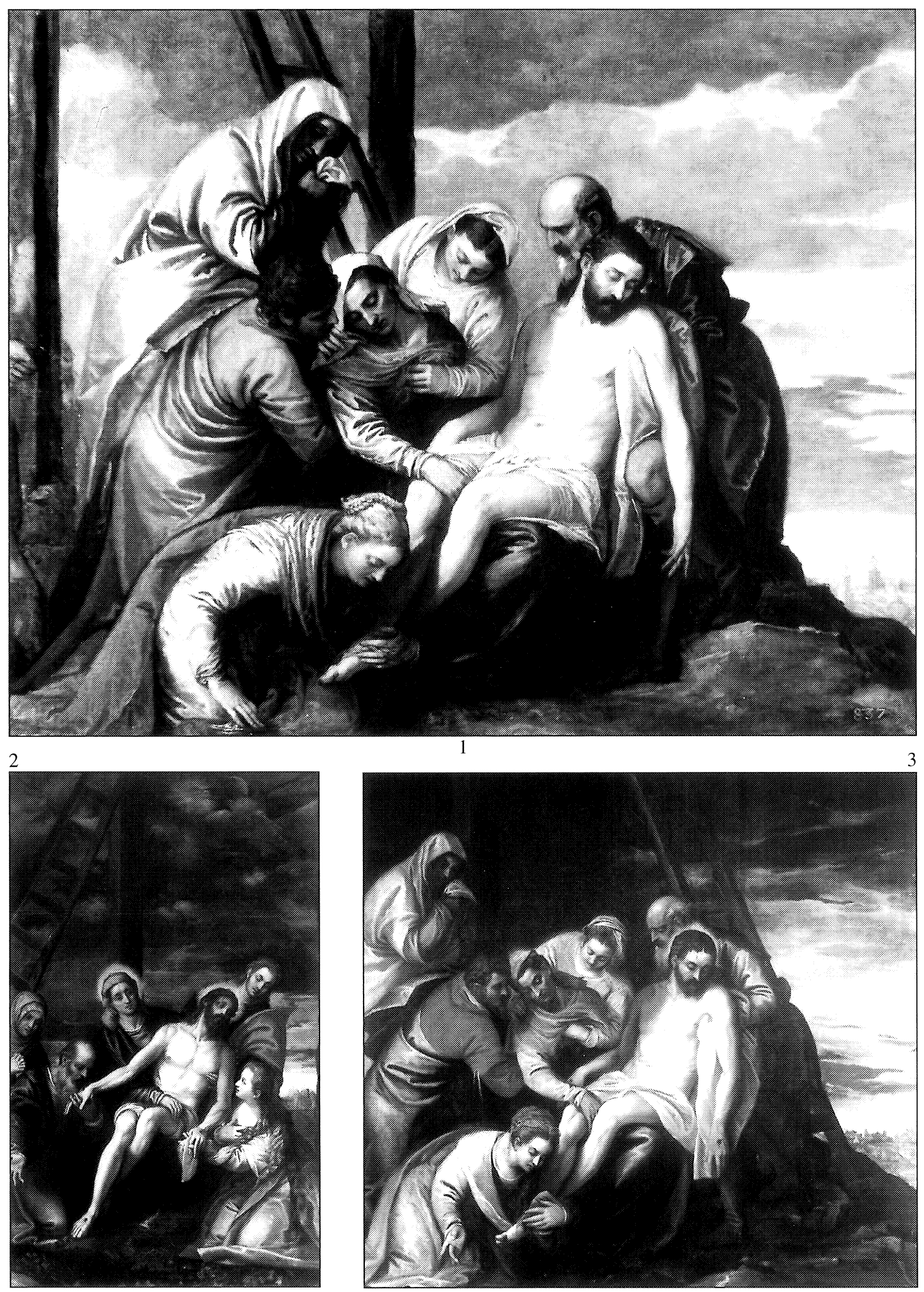

Fig. 1. Pablo Veronés: Descendimiento. Nuevos Museos.

Fig. 2. Carletto Caliari: Descendimiento. Panteón de Infantes.

Fig. 3. Carletto Caliari: Descendimiento. Nuevos Museos. El Escorial (Madrid), Monasterio (Fotografías cedidas y autorizadas por el Patrimonio Nacional). 
Soltura de pincelada, agilidad en el trazo, riqueza de colorido; todo en este hermosísimo cuadrito parece hablarnos de Pablo Veronés. El pincel traza de forma vibrante y quebrada los reflejos de las telas, ilumina con ligeros toques las carnaciones consiguiendo de esta manera impregnar la escena de viva palpitación. La paleta es suntuosa, clara, rica; el artista distribuye sabiamente los colores logrando que se potencien unos a otros y que se cree entre ellos una extraordinaria y sonora armonía; piénsese en el tierno verde del manto de San Juan rodeado por el vivo rojo de su túnica, el encendido naranja del manto de la Magdalena y el delicado amarillo crema del manto de la María que en pie llora compungida, la palidez del cuerpo de Cristo que resalta entre el rojo y el verde con los que se viste José de Arimatea y el brillante amarillo con reflejos naranjas de la túnica de la María que sostiene a la Virgen. El vigor que muestra en su colorido nos aleja de Carletto, quien siempre se valió de una gama de colores más sorda. Asimismo, su factura pictorista, deshecha y chispeante resulta muy distinta del gusto por las líneas claras y preciosistas de su hijo. En cuanto a su composición, me atrevería a decir que es la más hermosa que de este asunto ideó su autor: todas las figuras se encuentran perfectamente encajadas y relacionadas unas con otras; se han dispuesto de manera que quedan abrazadas por un círculo ideal, lo que aumenta su carácter íntimo. Si la comparamos con el cuadro del mismo asunto que se conserva en la Galleria Doria Pamphili ${ }^{7}$ — sin duda la composición más cercana a la del nuestro-, observaremos que gana a éste en rigor compositivo: en el cuadro romano el grupo de personajes que se encuentra detrás de la Virgen resulta algo forzado y no se integra armoniosamente con el resto; frente al sentido de bloque redondo y compacto de nuestra composición, la de la Galería Doria Pamphili tiende a la dispersión. Por otra parte, resulta sorprendente la similitud que presenta la María que de pie acerca su manto a la cara con su mano izquierda con la célebre «Magdalena» de Savoldo ${ }^{8}$, similitud que lleva a pensar que Veronés pudo conocer alguna de las versiones de dicha creación ${ }^{9}$. En cuanto a la

Abar». Vistos los documentos consultados por Burke, no ayuda mucho a clarificar su significado el cotejo de lo que se dice en el inventario (1688), en la tasación y en el documento de los cuadros de la almoneda del marqués del Carpio que pasaron a la corona (1691): en el primer documento se dice «bajo de abar» (Archivo Histórico de Protocolos, $\mathrm{n} .^{\circ} 9819-\mathrm{No}-$ tario Andrés de Calatañazor-, fol. 768v); en el segundo «vajado de Abar» (idem, fol. 1029v) y en el tercero «bajado de Abar» (idem, fol. 1179v). Asimismo, en la copia de la tasación conservada en el archivo de la casa de Alba (caja n. ${ }^{\circ} 221-$ 2) se dice «vajado de Abar». ¿Querrá, quizá, decir bajado del Calvario? En cambio, lo que sí resulta claro al leer los documentos del Archivo Histórico de Protocolos y el de la casa de Alba, es que Burke posiblemente se equivocara al transcribir «y Maria Con la Sauana Tendida», pues en los documentos señalados parece decir «y Mathia...» — que muy posiblemente sea una abreviación de Arimatea-, lo que correspondería a lo representado en el cuadro, pues, efectivamente, José de Arimatea sostiene con sus manos una sábana tendida.

7 Obra sobre la que la crítica no se ha manifestado unánime. Según Caliari (1888) y Fiocco sería autógrafa de Pablo Veronés, Berenson (1958) la consideró parcialmente autógrafa, Pallucchini (1958) obra de colaboración, Marini (1968) de Pablo Veronés con amplia colaboración y Pignatti (1976) se inclina a pensar que sea obra de Benedetto Caliari (Pignatti, T., Veronese. L'opera completa, Venecia, 1976, T. I, p. 205).

${ }^{8}$ Existen varias versiones autógrafas de esta creación, véase al respecto Cat: Exp. «Giovanni Gerolamo Savoldo tra Foppa, Giorgione e Caravaggio», Brescia, 1990, pp. 146-152 (fichas de catálogo de Renata Stradiotti de los ejemplares conservados en la Gemäldegalerie (Berlín), en una colección privada (Zurich) y en la National Gallery (Londres) y Frangi, F., Savoldo. Catalogo completo, Florencia, 1992, pp. 96-105-ejemplar que estuvo en una colección privada (Zurich), y los conservados en la National Gallery (Londres), en el Palazzo Pitti (Florencia) y en la Gemäldegalerie (Berlín)—.

${ }^{9}$ Nada tendría de particular que Veronés la conociera, pues se sabe que Savoldo residió en Venecia y es de suponer que muy probablemente existieran allí desde antiguo originales o copias de dicha creación. En 1648, Ridolfi al hablar de las obras de éste dice «in Brescia ... in casa Auerolda in vna figura della Maddalena inuolta in drappo, col vase dell'alabastro, incaminata al Sepolcro, celebre pittura, dalla quale si sono tratte molte copie ... Madama d'Ardier, Ambasciatrice Francese, haueua (en Venecia, se entiende) vn ..., \&vna delle Maddalene dette...» (Ridolfi, C., Le Maraviglie dell'Arte. Ovvero le Vite degli Illustri Pittori veneti e dello Stato, Venecia, 1648; edición von Hadeln, 1914, vol. I, pp. 271-272). Además, Veronés no la utilizó únicamente en la obra de El Escorial, sino que también lo hizo, aunque de una manera ligeramente menos evidente, en su «Crucifixión» del Louvre, cuya Magdalena considera Creighton Gilbert un homenaje de Veronés a Savoldo. El mismo Gilbert ha resaltado la influencia del pintor de Brescia en el de Verona, llegando a afirmar: «Se dobbiamo trovare un vereo grandissimo succesore all'isolato Savoldo, questo è un pittore vicino a Brescia, il Veronese» (Creighton Gilbert, «Savoldo cortese» en Cat. Exp. "Giovanni Gerolamo Savoldo tra Foppa, Giorgine e Caravaggio», Brescia, 1990, p. 46). 
fecha de realización de nuestro cuadrito habría que pensar que se trata de una obra de avanzados los años 70, ya que si por la cálida claridad de su colorido se relaciona con pinturas de dicha década — por ejemplo, las alegorías de «La Unión Feliz», «La Infidelidad», «El Desengaño» $\mathrm{y}$ «El Respeto» de la National Gallery de Londres o «La Anunciación» conservada en la Galleria dell'Academia de Venecia-, por su acentuado carácter íntimo, por reducir la representación a lo esencial y por su doloroso e interiorizado sentido de la piedad parece preludiar creaciones que la crítica suele situar a partir de 1580 (piénsese en la «Crucifixión» conservada en la Iglesia de San Lazzaro dei Mendicanti de Venezia, en el «Cristo muerto sostenido por la Virgen y un Ángel» del Museo del Ermitage de San Petersburgo o en el «Cristo en el Huerto sostenido por un Ángel» de la Pinacoteca Brera de Milán). Ya en el siglo XIX su estado de conservación debía dejar mucho que desear, pues en el mencionado documento de 14 de mayo de 1813, tras describirlo se dice: «estropeado». Hoy en día la situación no ha cambiado, pues se aprecian en varias partes pérdidas de pintura y repintes; obsérvese en particular el que groseramente se extiende por parte del manto de la María que figura de pie detrás de San Juan o el que cubre la tela que protege las piernas de la Virgen, tela que si hoy presenta un tono grisáceo, en un principio debería ser - a juzgar por lo que aparece en una pequeña parte en donde se ha desprendido el repinte - de un intenso azul ultramarino. En algún momento debió de ser recortado por su lateral izquierdo, ya que por allí asoman las pierna y una mano sosteniendo un clavo de un personaje que debió formar parte de la composición.

De los otros dos «Descendimientos» el padre Ximénez parece referirse al que se conserva hoy en el Panteón de Infantes (Fig. 2) cuando cita en la Celda Alta del Prior: «Enmedio de la Banda de Poniente... Corresponde á este (se refiere a la «Resurrección de la hija de Jairo» de Girolamo Muziano, cuadro de dimensiones muy semejantes al «Descendimiento» del Panteón de Infantes ${ }^{10}$ ) un Descendimiento tambien grande, de seis Figuras (en el conservado en el Panteón de Infantes son seis los personajes representados, mientras que en el otro son siete) del natural, distribuidas con arte: pintura magestuosa, labrada por Carlos Caliari» ${ }^{11}$. Ponz lo verá en el mismo lugar ${ }^{12}$. Es muy posible que la obra vista por Ximénez y Ponz corresponda a la mencionada por el padre Santos en el Oratorio de la Celda Alta del Prior: «El Altar està en el Testero defrente de la Ventana; siempre vestido, y compuesto, como los de la Iglesia. El Retablo es vn Quadro grande, del Descendimiento de la Cruz, de mano de Carlos Veronense, y que se le conoce la mano, en lo primoroso de la execucion» ${ }^{13}$.

En el siglo XIX, contamos con referencias a ambos «Descendimientos». Bermejo menciona en el Claustro Principal Alto el hoy conservado en los Nuevos Museos: «1. El Descendimiento de la Cruz. Componen el historiado María Santísima y otras tres mugeres, san Juan y José Abarimatea: figuras del tamaño natural, de escuela Veneciana, y parece de Cárlos Veronés» ${ }^{14}$,

\footnotetext{
10 Poleró facilita las siguientes medidas del cuadro de Muciano: «Alto, 9 piés, 11 pulg., 10, lín.; ancho, 6 piés, 2 pulg., 4 lín.» (op. cit., 1857, p. 50, cuadro n. ${ }^{\circ} 112$ ); en cuanto al «Descendimiento» hoy en el Panteón de Infantes: «Alto, 9 piés, 10 pulg., 7 lín.; ancho, 6 piés, 1 pulg., 11 lín.» (op. cit., 1857, p. 50, cuadro n. ${ }^{\circ} 113$ ).

11 Ximénez, A., Descripción del Real Monasterio de San Lorenzo del Escorial, Madrid, 1764, p. 125.

12 «un Descendimiento de la Cruz, en figuras del natural, de Carlos Cagliari» (Ponz, A., Viage de España, Madrid, 1788, T. II, Carta IV, n. ${ }^{\circ} 77$; Madrid, Edit. Aguilar, 1988, vol. I, p. 409).

${ }_{13}$ Santos, Francisco de los, Descripción breve del Monasterio de S. Lorenzo El Real del Escorial, Madrid, 1657 , fol. 70a. En la edición de 1667 se cita en el mismo lugar, fol. 86v. Por la edición de 1681 se sabe, como bien puso de manifiesto Checa (ver nota a pie de página $n .{ }^{\circ} 1 \mathrm{del}$ presente artículo), que se había sustituido por un «nueuo Retablo de Talla, labrado con mucha diferencia, y adorno, y en medio vna Imagen de Nuestra Señora de la Concepcion hermosissima, de Escultura admirable, en trono de Angeles, que causa grande amor, y deuocion» (fol. 71a). En la misma edición de 1681, al hablar de la Celda Alta del Prior no se detallan las pinturas que allí se encuentran, con lo cual resulta posible que ya hubiera sido trasladada allí y que, años después, la viera Ximénez en dicho lugar.

No hay posibilidad de que se trate del cuadro n. ${ }^{\circ} 85$ del cat. Poleró, pues por sus medidas $($ L. $69,5 \times 89)$ no puede considerarse «vn Quadro grande».

${ }^{14}$ Bermejo, D., op. cit., Madrid, 1820, p. 226.
} 
lugar en donde lo vio también Poleró ${ }^{15}$. Por lo que respecta al conservado en el Panteón de Infantes, tiene que ser el que cita el mismo Bermejo en el Aula del Moral, pues es sin duda el catalogado por Poleró en dicho lugar ${ }^{16}$.

Respecto al «Descendimiento» grande conservado en los Nuevos Museos (Fig. 3) ${ }^{17}$, aunque su composición se base en el cuadrito de Pablo Veronés que se acaba de estudiar, no puede considerarse una copia de éste. Pocas son las diferencias en las posturas de sus personajes; sin embargo, su estructura es muy distinta. Mientras, como se ha dicho, las figuras del cuadrito se dirían inscritas en un círculo, en el caso del lienzo que nos ocupa se ordenan en un cuadrado. A ello contribuye el simple hecho de erguir algo más tanto la figura de José de Arimatea como la de la María que cierra la composición por su lado izquierdo. Las vestimentas de algunas figuras coinciden en su colorido, aunque no en el tono elegido. En términos generales, se podría decir que la paleta utilizada es más pálida, más apagada; los colores aparecen, como dice Crosato Larcher al hablar del primer Carletto, «impastati di una luce grigiastra di riminiscenza bassanesca» ${ }^{18}$. Por otra parte, siempre con respecto al cuadrito, se han simplificado los pliegues y se ha puesto en evidencia sobre todo su carácter lineal. Frente a la pincelada viva y nerviosa de aquél, predomina en éste una amplia y serena manera, propia de Carlo Caliari, de conducir el pincel a través del cuadro. Asimismo, se han cargado las sombras con lo que se ha oscurecido la escena adquiriendo la atmósfera un marcado carácter crepuscular.

En cuanto al «Descendimiento» que se encuentra en el Panteón de Infantes ${ }^{19}$, su composición se relaciona muy estrechamente con la del cuadro homónimo conservado en la iglesia de l'Annunziata de Ostuni ${ }^{20}$. Entre ambas composiciones se aprecian pocas diferencias, que pueden resumirse de la siguiente manera: en la de Ostuni no existe la figura de la mujer con las manos unidas en signo de oración que aparece tras José de Arimatea en la de El Escorial; en ésta la cabeza de Cristo se encuentra inclinada hacia atrás, mientras que en aquella cae ladeada hacia la izquierda; aunque ambas Magdalenas estén de rodillas y tengan las manos en posición semejante, la de El Escorial mira al rostro de Cristo, mientras que la de Ostuni tiene sus ojos clavados en su mano izquierda; asimismo, los angelitos que aparecen en la Cruz de esta última, no existen en aquélla. Dentro de la carrera de Carletto, el lienzo de El Escorial debiera situarse en su época de plena madurez, época en la que, según Crosato Larcher: «ritorna ai moduli di paolo e li ricrea personalmente, ma cade nell'enfatico: egli non comprende il notturno tardo del padre e quindi schirisce, e cerca colori tenui... tuttavia non si può negare una fastosa imponenza a queste opere e una notevole luminositá» ${ }^{21}$. Clara resulta la referencia al mundo paterno, pues tanto su composición, que se basa en un dibujo suyo, como la fisonomía de los personajes nos hablan de Pablo Veronés. Sin embargo, tiene la obra un carácter decla-

15 «Calos Veronés. 189 El Descendimiento. La Virgen traspasada de dolor y acompañada de las Marías, recibe en su regazo el sagrado cuerpo del Redentor. Alto, 6 piés, 6 pulg., 6 lín.; ancho, 7 piés» (Poleró, V., op. cit., 1857, p. 65).

16 «8. El Descendimiento de la Cruz, por Carlos Veronés» (Bermejo, D., op. cit., Madrid, 1820, p. 237); «Carlos Veronés. 113 El Descendimiento de la Cruz. La Virgen, al pie del madero santo, sostiene en su regazo el cuerpo muerto de su sagrado Hijo; San Juan y las Marías lloran su muerte. Fondo, la ciudad santa. (Firmado). Alto, 9 piés, 10 pulg., 7 lín.; ancho, 6 piés, 1 pulg., 11 lín.» (Poleró, V., op. cit., 1857, p. 50)

${ }_{17}$ En su ángulo inferior izquierdo figura el n. ${ }^{\circ} 189$ en blanco (n. ${ }^{\circ}$ del catálogo de Poleró).

${ }_{18}$ Crosato Larcher, L., «Per Carletto Caliari», Arte Veneta, 1967, año XXI, p. 110.

19 En su ángulo inferior izquierdo se lee: «Carlo caliari veronese».

No tengo noticia de que recientemente esta pintura haya sido objeto de un estudio crítico. Ahora bien, en El Escorial. La octava maravilla, figura reproducida a nombre de nuestro pintor (1987, lám. 136) y Martínez Cuesta, J. la cita también como obra de Carlo Veronés (Guía del Monasterio de San Lorenzo el Real también llamado de El Escorial, 1992, p. 110).

${ }^{20}$ Obra que se encuentra en muy mal estado de conservación y que la mayor parte de la crítica ha considerado autógrafa de Pablo Veronés (Berenson, 1958; Palluchini, 1958 y 1984; Cocke, 1973; Pignatti y Pedrocco, 1991). Sin embargo, Valconover (1979) la consideró obra de taller, opinión compartida en 1995 por Pignatti y Pedrocco. Como puso de manifiesto Cocke (1984) su composición se basa en un dibujo de Pablo Veronés conservado en Berlín (cf. Pignatti, T. y Pedrocco, F., Veronese. L'opera completa, Milán, 1995, T. II, p. 518), dibujo en el que también se basa nuestra «Deposición».

${ }^{21}$ Crosato Larcher, L., art. cit., p. 113. 
matorio que nos aleja de la elegante y acompasada manera de narrar del padre. A pesar de la atmósfera crepuscular que impregna la escena, Carletto no oscurece su paleta, con lo que confiere al cuadro una cierta extraña luminosidad. Asimismo, se vale de un cierto claroscurismo de raíz bassanesca. Con esta obra demuestra nuestro pintor algo de lo que ha aprendido de su padre y del taller de los Bassano: de aquél a componer con cierta majestad y grandeza y de éste a poner más al alcance del espectador lo representado.

José María Ruiz Manero

Doctor en Historia del Arte

\section{HALLAZGO DEL PRIMER CUADRO EXPUESTO POR RAIMUNDO DE MADRAZO}

Queremos presentar en este trabajo un cuadro del pintor Raimundo de Madrazo sobre el que la bibliografía del artista ha hecho mención en numerosas ocasiones, por tratarse del primer cuadro que dicho artista presentó en una exposición; fue realizado a la temprana edad de dieciséis años. Es un óleo sobre lienzo, de $130 \times 221$ centímetros firmado en el ángulo inferior izquierdo: «R. Madrazo/1859». Representa La traslación de los restos del apóstol Santiago a la sede de Padrón. El cuadro fue adquirido en 1906 en Sevilla, y desde entonces ha permanecido en la misma colección particular. Se conserva un documento que certifica el origen del mismo y se pagaron 1.000 pesetas por su importe. En el momento de su compra, los profesores de la Escuela de Bellas Artes de Sevilla, Fernando Tirado y Francisco Anaya y León ratificaron la autoría de mano de Raimundo de Madrazo, al igual que el pintor Alfonso Cañaveral, que además añade el dato de que Cecilia de Madrazo posó para la figura del ángel y que el mismo Fortuny pintó el personaje que aparece en el fondo levantando los brazos. También ratifica la autenticidad de la firma J. García y Ramos, que al rubricar los apellidos con la sola inicial de su nombre, nos plantea la interrogante de saber si se trata de José o de su hermano Juan. Nos inclinamos a pensar que es Juan, porque no adorna su nombre con los títulos de presidente de la Academia Libre de Bellas Artes (nombramiento que se le hizo tras su vuelta del extranjero en 1881) ${ }^{1}$ o el de académico de Bellas Artes (que disfrutó José desde 1893) ${ }^{2}$; además, si comparamos la rúbrica del documento con alguna de las que se aprecian en reproducciones de sus cuadros podemos notar diferencias. Y nos consta por otro documento, conservado en la misma colección, que dos años antes de la compra del lienzo de Madrazo se habían adquirido otros cuadros en Sevilla, concretamente ocho cobres flamencos que fueron comprados en 1904 a don Juan García y Ramos, lo que demuestra una relación comercial de continuidad de este pintor con el mismo coleccionista.

En el mismo certificado de 1906 el restaurador Manuel Fatuarte ratifica el perfecto estado de conservación de la obra. Siendo bien conocidos en el ámbito sevillano tres de los firmantes de este documento, Fernando Tirado, Alfonso Cañaveral y García Ramos, no tenemos datos acerca de Francisco Anaya y León ni de Manuel Fatuarte, aunque de éste sí sabemos de sus labores de restauración y valoraciones para la misma colección e incluso se conserva en ella una pequeña obra original suya, una vista arquitectónica por él rubricada.

\footnotetext{
1 Quesada, L., La vida cotidiana en la pintura andaluza, Focus, Sevilla, 1992, p. 196.

${ }^{2}$ Valdivieso, E., Historia de la pintura sevillana. Siglos xIII al XX, Guadalquivir, Sevilla, 1986, p. 427.
} 